\title{
THE ROLE OF MECHANICAL FORCE IN MOLECULAR AND CELLULAR DURING ORTHODONTIC TOOTH MOVEMENT
}

\author{
Ida Bagus Narmada, Achmad Syafei \\ Department of Orthodontic Faculty of Dentistry Airlangga University \\ Department of Orthodontics, Faculty of Dentistry Airlangga University Surabaya Indonesia.
}

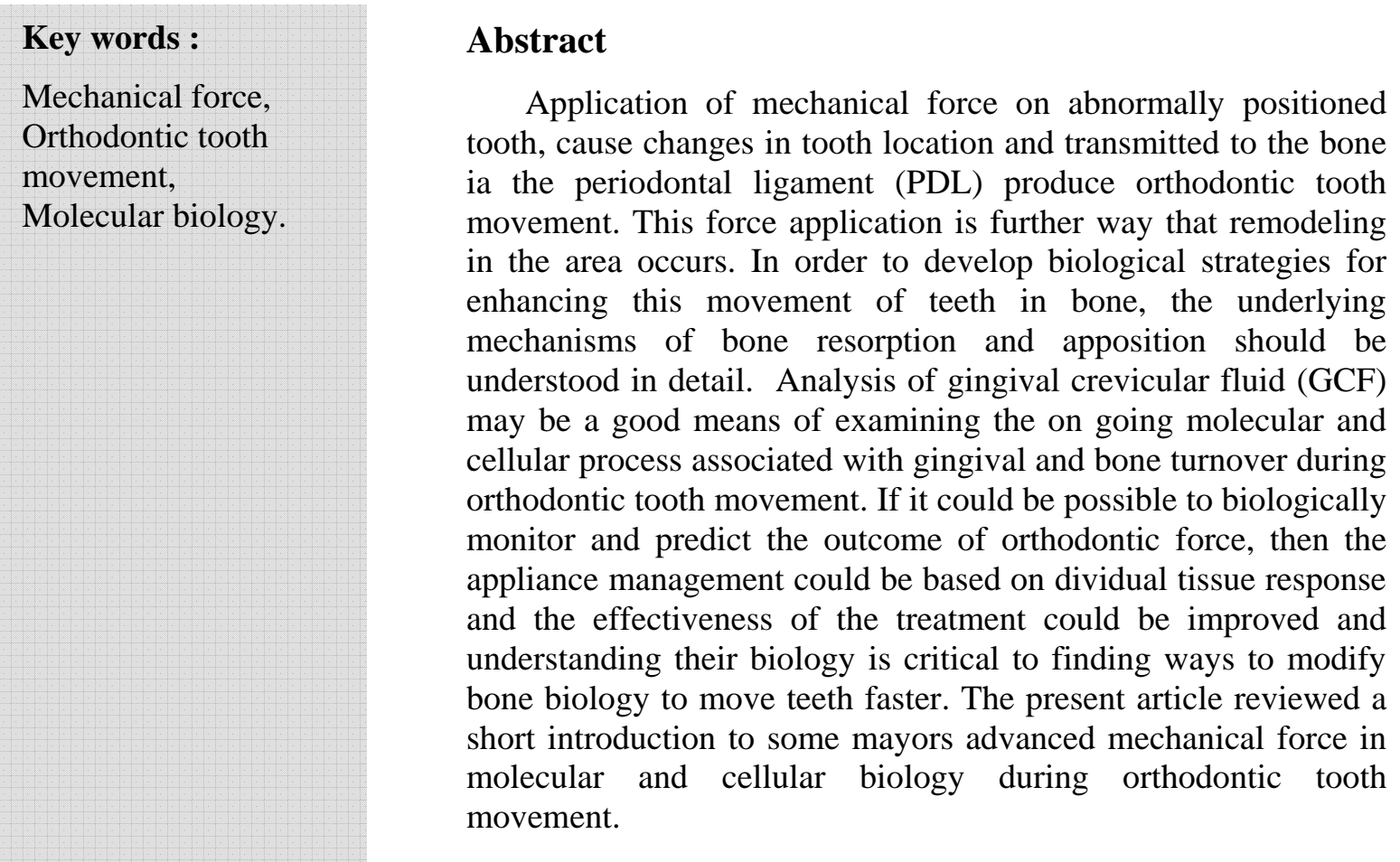

\section{Introduction}

In clinical orthodontic treatment requires application of force system to individual teeth or groups of teeth, which result in cellular response with periodontal ligament (PDL) and alveolar bone remodeling. The force applied must be off sufficient magnitude and duration to exceed the normal physiologic threshold associated with daily oral function. Excessive force levels will result in area of tissue necrosis 
with delayed tooth movement and increased risk of root resorption. The PDL, the soft tissue matrix that joins the dental root to the alveolar bone, is composed of an intricate network of blood vessel and nerve ending, the principal cellular components of which are fibroblast and progenitor cells. $^{(1)}$

Although orthodontic tooth movement is achieved in large segment of population, the optimum force level has not been defined. The optimum force for tooth movement depends on individual root geometry as well as biologic characteristics of surrounding tissue including bone density, periodontal thickness, and dynamics of gingival crevicular fluid (GCF) which has three sources : cellular, vascular, and interstitial. ${ }^{(2,3,19)}$

The current concept of optimal force is base on the hypothesis that a force of a certain magnitude and temporal characteristics continuous, intermittent, constant and declining would be capable of producing a maximum rate of tooth movement without tissue damage and with maximum patient comfort. The optimal force for tooth movement may differ for each individual patient Orthodontic appliance is generally adjusted or activated about once per month. Within several days after the appliances is adjusted and sustained force is applied to the teeth, some patients complain of senses of in congruity of teeth. It has been reported that such an usual sensations are diminished after one or two weeks. ${ }^{(4)}$ It has been an important matter of concern to examine the effect of orthodontic force toward the periodontal tissue, especially, cellular and molecular during tooth movement.

\section{Tooth, Periodontal Ligament, and Alveolar} Bone

The teeth are both secured and suspended within their bony alveolar sockets by a fibro vascular PDL. The PDL consist of cellular components and intercellular components, which fill up the space between the cells. Intercellular components, i.e. extra cellular matrix (ECM), are mainly composed of fibrous elements and ground substance. ${ }^{(5)}$ The mayor fibrous elements are collagen fibers type I and type III, which play a role in resisting tensional force and holding teeth in the alveolar socket. ${ }^{(6)}$ The mayor components of the ground substance of the PDL are proteoglycans (core protein + long chain glycosaminoglycans) and glycoproteins. There are considered to contribute to viscoelastcity and are necessary in the process of remodeling the PDL. It is thought that proteoglycan production and distribution change in response to mechanical stress on tissue. ${ }^{(6,7)}$ Cells of the PDL include osteoblast, osteoclast, fibroblasts, epithelial cell rest of Malassez, macrophage, undifferentiated mesenchymal cell and cementoblasts. Fibroblasts are the principal cells of PDL and are rich in bone alkaline phosphatase activity and osteokalsin. As this enzyme is a requisite for phosphate metabolism in the mineralization of bone and cementum formation, the PDL fibroblasts are thought to play a role in alveolar bone metabolism. ${ }^{(8,12)}$

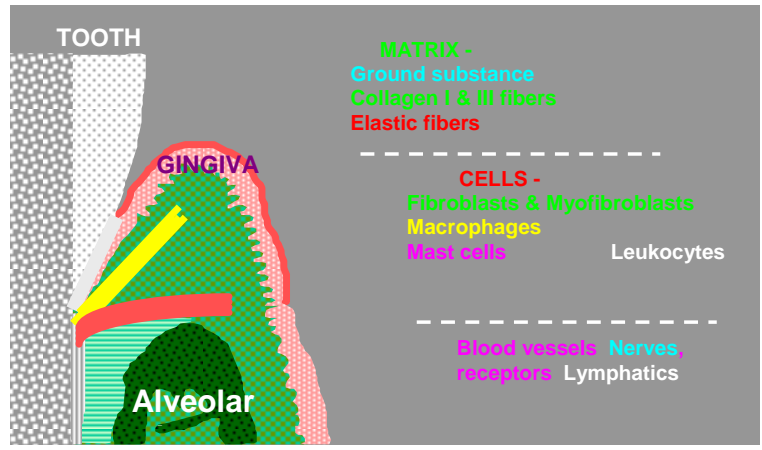

Figure 1. Tooth, Periodontal Ligament, and Alveolar Bone

The purpose of an orthodontic appliance is to apply a mechanical force directly to a tooth with the force vector aimed in the intended path or direction of tooth movement. Many devices have been used over the years, with varying degrees of success, but all rest upon the same principle that if a sufficient force is applied directly to a tooth, the surrounding PDL and alveolar bone will continue to remodel and adapt until such time that the force is withdrawn either by removal of the appliance or once the tooth moved a sufficient distance such that the original force stored in the wire or spring of the appliance has dissipated.

When the force $(\mathrm{F})$ is applied to the tooth, the associated fibrous and cellular components of the PDL have some capacity to distort as an 
immediate response. The simplest model is a single rooted tooth like a mandibular canine. The PDL on the side which the force is directed toward will become compressed. This called the pressure side. The PDL on the side which the force is directed away from will become elongated. This called the tension side. This model and the concepts of pressure side and tension side should be kept well in mind as establish a natural conceptual division between the two simultaneous processes which seem necessary in order for teeth to move.

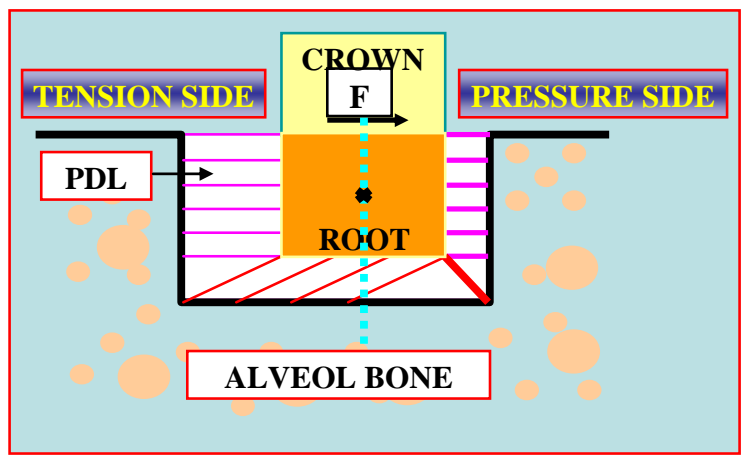

Figure 2. Mechanisms of orthodontic tooth movement

Orthodontic forces produce a distortion of the PDL matrix resulting in an alteration in cellular shape and cytoskeletal configuration, and neuropeptide release from afferent nerve endings. At the biomolecular level, these forces may induce the release of prostaglandins, growth factors, and cytokines. ${ }^{(9,10)}$

Histochemical, immunohistochemistry (IHC) on animals and biochemical studies on human beings support this conclusion. Finally orthodontic forces appear to generate cytokines that effect the formation and resorption of bone. One such cytokine, tumor necrosis factors - alpha (TNF), is of particular importance since has been shown to be an early modulator of bone resorption and is detectable in the human GCF. Deposition of new osteoid on the tension side began after 3 days of treatment, area of active osteoclastic resorption with multinucleated osteoclast in their lacunae were found in the pressure side after 4 days of treatment, marker gene expression for osteoblastic differentiation includes: alkaline phosphatase, osteocalcin, and bone sialoprotein all detected by IHC. Proliferation and recruitment of osteoblast precursors is an important step in osteogenesis on periosteal surfaces, and increase in the number of osteoblasts in the area of otherwise intense bone matrix synthesis suggests that, during bone formation, proliferation of cell has a smaller role compared to the marked increase in differentiation individual cell. ${ }^{(11,12)}$

\section{Biology of Bone Remodeling and Root Resorption}

Due to the low level of forces applied throughout the treatment, the microcirculation at and around the root surface is not compromised. Two biological phenomena need to be described, one of the alveolar bone remodelling and the other integrity of the root structure. In terms of remodelling, a modified type of the alveolar bone resoption is observed. In the traditionally ligated system, where force levels applied and necessary for the tooth movement were in the range of several hundred grams of force, the orthodontic movement was typically described in three phases : (1) The initial movement due tot he PDL compression, (2) The hyalinisation period within the PDL with very little or no tooth movement, (3) The tooth movement caused through the process of undermining resorption and largely mediated by the presence of oxygen, brought about by reopening of the capillary network..$^{(4,13)}$

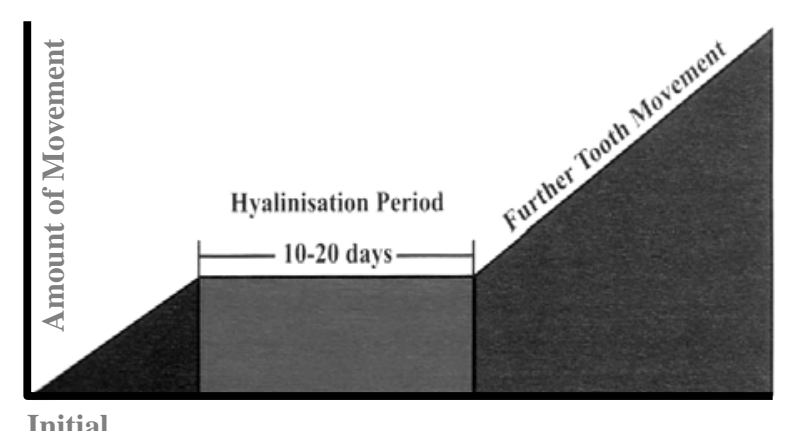

Initial

Figure 3. These stages can be diagrammatically shown in this fashion

Ideally, the clinician would want to eliminate the second phase [shown in red], because it is not a biologically positive or 
clinically advantageous stage. Instead, one would want to move the tooth in an uninterrupted fashion, where the line representing the tooth movement would be continually advancing in an upward direction with the positive slope and without any significant plateaus or interruptions of that trend. This would additionally suggest the presence of predominantly the desired, frontal bone resorption. With the application of low, continuous forces the tooth movement can appear to be uninterrupted and quite different from that shown on the diagram. It is nearly continuous. More precisely it fluctuated in cycles of approximately two days. This process is characterized primarily by the frontal resorption on the pressure side of the root, where the oxygen supply is not seriously compromised, thus allowing osteoclastic activity adjacent to the PDL. At the same time, the tension side is preparing for, and then within a few days starts laying down, the new osteomaterial. With this synchronous bone formation and resorption, a new or remodelled alveolled alveolar socket is formed, contributing to the apparent fluid motion of fluid mechanics of the tooth movement. The root structure responds in a different fashion, because the root resorption is veiwed by most as a negative and undesirable sequel of orthodontic treatment. Among various factors possibly responsible for the root resorption, the ones often listened as the most likely are force level and duration. The light, continuous forces typically cause neither pain for the patient nor root resorption. Also, because of the shorter duration of active treatments with interactive self-ligation, forces are applied for a shorter time, further reducing the likelihood of the root resorption. ${ }^{(14,15,16,17,21)}$

\section{Discussion}

The tooth-supporting tissue are constantly remodeled under ormal conditions with physiological tooth migration. With orthodontic intervention prolonged pressure on the teeth result in enhanced remodeling of periodontal structures, including supracrestasl gingival and PDL fibers, as well as alveolar bone. Study of orthodontically induced reactions in the human PDL has been limited. Knowledge of historical responses in the supporting structures of teeth during orthodontic tooth movement is mainlybased on animal studies. It has been suggested that the initial phase of orthodontic tooth movement involves many inflammatory-like reactions within the periodontal tissues, characterized by vascular changes and migration of leukocytes out of PDL capillaries. ${ }^{(17,18,21)}$ The remodeling involves differentation of resident PDL cells into osteoblasts and fibroblasts. ${ }^{(1,4,6)}$

Osteoblasts control both the resorptive and formative phases of the bone remodeling cycle by regulating osteoclasts recruitment and activity. In addition, osteoblasts produce collagenases, which decgrade nonmineralized osteoid covering the resting bone surface, and thus enable the osteoclasts to gain access to the mineralized tissue. Prostaglandin (PG) functions as a mediator of the bone resorption induced by experimental tooth movement. ${ }^{(8,9,10,12)}$

On the pressure side, osteoclasts attact the alveolar bone from the PDL space in the process of direct resorption, allowing rapid tooth movement. On the tension side, new bone is deposited by the osteoblasts until the width of the PDL space has returned to its normal limits. Overcompression in limited areas of the PDL frequently totally occludes blood flow, causing cell death and the development of a cell-free necrotic area. In light microscopy, the tissue reveals a glass like appearance termed hyalinization. When hyalinization occurs, osteoclasts differentiate from the cells within the adjacent bone-marrow spaces to start undermining resorption from the underside of the lamina dura. This undermining resorption results in a inevitable delay in tooth movement and may increase root resorption. ${ }^{(15,21,22)}$

GCF is a complex mixture of substances derived from serum, host inflammatory cells, structural cells of the periodontium, and oral bacteria. GCF originates from the vessels of the gingival plexus of blood vessels and flows through the external basement membrane and the junctional epithelium to reach the gingival sulcus. GCF can be isolated from health sulcus, although only in small amounts. In the healthy 
periodontium, GCF represents the transudate of gingival tissue interstitial fluid produced by an osmotic gradient. The volume of GCF increases during periodontal inflammation, primarly due to an increase in the permeability of the vessels underlying the junctional and sulcular epithelium, and also due to basement membrane changes. In addition, the enlarged intercellular spaces of the junctional epithelium act as a reservoir for GCF. At the same time, its composition starts to resemble that of an inflammatory exudate. ${ }^{(2,3,19)}$

The collection and analysis of GCF have provided a non-invasive and site-specific means to assess the biochemical status of the marginal periodontium. GCF composition is well documented as reflecting also the metabolic state of the deep-seated tissues of the periodontium, e.g., alveolar bone turnover. ${ }^{(13,14)} \mathrm{A}$ considerable number of bacteria and hostderived products found in the GCF have been associated with the initiation and progression of periodontal disease. Biochemical analysis of GCF shows promise as an effective means for early detection of periodontal disease. In order to monitor non-invasively the expression of biologically active substances in humans, changes have been studied in the composition of GCF, elevation and partial activation of mutiple species of polymorphonuclear leukocyte (PMN) and fibroblast-type MMP reflect periodontal remodelling during orthodontic tooth movement. ${ }^{(18,20,21)}$

\section{Reference}

1. Jenifer J. Lowney, DMD, Louis A. Norton, DMD, David M. Shafer, DMD, and Edward F. Rossomando, DDS, PhD., Orthodontic Forces Increase Tumor Necrosis Factor A In The Human Gingival Sulcus, $J$ Am. Orthod Dentofac Orthop, p 1995; 108 : 519-24.

2. Yijin Ren, DDS, MSc, Jaap C. Maltha, PhD, Anne Marie Kuijpers-Jagtman, DDs, PhD. Optimum Force Magnitude for Orthodontic Tooth Movement : A Systematic Literature Review, Angle Orthodontics 2003; 73: p. 86-90.

3. Bien MS., 1983, The Dynamics of Periodontal Fluid Composition. Arch Oral Biol, p. 304 : 123-42.
4. Proffit WR., 1999, Contemporary Orthodontics, St. Louis, Calif : Mosby-Year Book Inc, p. 296 - 325.

5. Pollanen MT, Salonen Jl, Uitto V-J, 2003, Structure And Function Of The Tooth-Epithelial Interface in Health and Disease, Periodontol 2000, p. $31: 12-31$.

6. Kaneko S., Ohashi K., Soma K., Yanagishita M., , Occlusal Hypofunction Causes Changes In The Proteoglycan Content In The Rat Periodontal Ligament, J. Periodont Res 2001;36,p. 9-17.

7. Beertsen W., Holmbeck K., Niehof A., Bianco P., Chrysovergis K., Birkedal-Hansen $\mathrm{H}$, et al, On the role of MT1-MMP, A Matrix Metalloproteinase Essential To Collagen Remodeling, In Murine Molar Eruption And Root Growth. Eur J Oral Sci 2002, p. 110 : 445 -51 .

8. Isik F., Sayinsu K., Arun T., Unlucerci Y., , Bone Marker Levels In Gingival Crevicular Fluid During Orthodontic Instrusive Tooth Movement : A Preliminary Study, The Journal of Contemporary Dental Practice 2005; Vol. 6, No. 2.

9. McColloch CAG, Nemeth E., Lowenberg B., Melcher AH., 1987, Paravascular Cells In Endosteal Spaces Of Alveolar Bone Contribute To Periodontal Ligament Cell Populations. Anat Rec, p. $219: 233-42$.

10. Canlis E., McCarthy TL, Centrella M., 1991, Growth Factors And Cytockines In Bone Cell Metabolism. Ann Rev Med, p. 42 : 17 - 24.

11. Kaneko S., Ohashi K., Soma K., Yanagishita M., Occlusal Hypofunction Causes Changes In The Proteoglycan Content In The Rat Periodontal Ligament, J. Periodont Res 2001; 36, p. 9 - 17.

12. Pavlin D., Magness M., Zadro R., Goldman ES., Gluhak-Heinrich J., 2000, Orthodontically Stressed Periodontium Of Transgenic Mouse As A Model For Studying Mechanical Response In Bone : The Effect On The Number of Osteoblasts, Clin Orthod. Res. 3, p. 55 - 66.

13. Reitan K. Clinical And Histologic Observations On The Tooth Movement During And After Orthodontic Treatment. Am J Orthod, p 1967; 53 : $721-45$.

14. King GJ., Fishlshweiger W , The Effect Of Force Magnitude On Extractable Bone Resorptive Activity And Cemental Cratering In Orthodontic Tooth Movement. J Dent Res., $p$ 1982; 61 : 775 - 9 .

15. Brudvik P, Rygh P, Non Clasts Cells Start Orthodontic Root Resorption In The Periphery Of Hyalinized Zones. Eur J Orthod, p 1993; 15 : $467-80$. 
Ida Bagus Narmada, Achmad Syafei

16. Bonafe-Olivera L, Faltin RM, Arana-Chavez VE, Ultrastructural And Histochemical Examination Of Alveolar Bone At The Pressure Ereas Of Rat Molars Submitted To Continuous Orthodontic Force. Eur J Oral Sci, p 2003 ; $111: 410-6$.

17. Redlich M., Reichenberg E., Harari D., Zaks B., Sholsham S., Palmon A , The Effect Of Mechanical Force on mRNA Levels Of Collagenase, Collagen Type I And Collagen Type I, And Tissue Inhibitors Of Metalloprotein ases In Gingivae Of Dogs. J Dent Res, $p$ 2001; $80: 2080-4$

18. Redlich M., Roos H., Reichenberg E., Zaks B, Grosskop A., Bar Kana I., Pitaru S., Palmon A., 2004, The Effect of Centrifugal Force On mRNA Levels Of Collagenase, Collagen Type-I, Tissue Inhibitors of Metalloproteinases And Beta-Actin In Cultured Human Periodontal Ligament Fibroblasts, J Periodontal Res, p. 39 (1) : $27-32$.
19. Alfano MC, 1997, The Influence Of SexChromosome Genes On Tooth Size In Man. A Genetic And Quantitative Study, Proc Finn Dent Soc, p. $67: 1-54$.

20. Ignman T, Apajalahti S, Mantyla P, Savolainen P., Sorsa T., Matrix Metalloproteinase-1 and -8 In Gingival Crevicular Fluid During Orthodontic Tooth Movement : A Pilot Study During 1 Month Of Follow-Up After Fixed Appliance Activation, Eur J Orthod, p 2005; 27 (2) : 2002 $-7$.

21. Mladen M. Kuftinec, DMD, ScD, M.Maija Eltz, Dr. Med. Univ., 2005, Interactive Self-Ligation System In-Ovation-R. GAC International, Inc., p $5-8$.

22. Rygh $\mathrm{P}$, Ultrastructural Changes Of The Periodontal Fibers And Their Attachment In Rat Molar Periodontium Incident To Orthodontic Tooth Movement. Scand J Dent Res 1974; 82 : $467-80$. 